\title{
Neurophysiological Characterization of Attentional Performance Dysfunction in Schizophrenia Patients in a Reverse-Translated Task
}

\author{
Jared W Young ${ }^{*, 1,2,5}$, Andrew W Bismark ${ }^{2,5}$, Yinming Sun ${ }^{3,4}$, Wendy Zhang', Meghan Mcllwain ${ }^{1,6}$, \\ Ibrahim Grootendorst ${ }^{1,7}$ and Gregory A Light ${ }^{1,2}$ \\ 'Department of Psychiatry, University of California San Diego, La Jolla, CA, USA; ${ }^{2}$ Research Service, VA San Diego Healthcare System, San Diego, \\ CA, USA; ${ }^{3}$ Temerty Centre for Therapeutic Brain Intervention, Centre for Addiction and Mental Health, Toronto, Ontario, Canada; ${ }^{4}$ Institute of \\ Biomaterials and Biomedical Engineering, University of Toronto, Toronto, Ontario, Canada
}

\begin{abstract}
Attentional dysfunction in schizophrenia (SZ) contributes to the functional deficits ubiquitous to the disorder. Identifying the neural substrates of translational measures of attentional dysfunction would prove invaluable for developing therapeutics. Attentional performance is typically assessed via continuous performance tasks (CPTs), though many place additional cognitive demands with little cross-species test-relevance. Herein, event-related potentials (ERPs) were used to investigate the neurophysiological correlates of attention and response inhibition of SZ and healthy participants, whereas they performed the cross-species-translated five-choice CPT (5C-CPT). Chronically ill, medicated SZ patients and matched controls ( $n=25$ SZ and 26 controls) were tested in the 5C-CPT, in conjunction with ERP and source localization assessments. The ERPs generated in response to correctly identified target and non-target trials revealed three peaks for analysis, corresponding to sensory registration $\left(P_{1}\right)$, response selection $\left(N_{2}\right)$, and response action $\left(P_{3}\right)$. Behavioral responses revealed that $S Z$ patients exhibited impaired attention driven by impaired and slower target detection, and poorer cognitive control. ERPS revealed decreased $N_{2}$ amplitudes reflecting poorer response selection for both target and non-target trials, plus reduced non-target $P_{3} s$ in SZ patients, the latter accounting for $37 \%$ of variance in negative symptoms. Source analyses revealed that the brain regions of significant differences localized to the left dorsolateral prefrontal cortex during response selection and the posterior cingulate cortex for cognitive processes. SZ patients exhibited impaired attention and cognitive control, characterized by less robust frontal and parietal ERP distributions across the response selection and cognitive response time windows, providing neurophysiological characterization of attentional dysfunction in SZ using the reverse-translated 5C-CPT.
\end{abstract}

Neuropsychopharmacology (2017) 42, I338-1348; doi:I0.1038/npp.2016.268; published online 8 February 2017

\section{INTRODUCTION}

Patients with schizophrenia (SZ) exhibit poor cognitive performances across numerous domains, which correlate with their ability to live independently (Green, 1996; Green et al, 2008). Considering current treatments predominantly target only positive symptoms of the illness, with limited-tono efficacy for treating these disabling cognitive deficits (Keefe et al, 2007; Parks et al, 2008), research has been galvanized toward identifying procognitive treatments for $\mathrm{SZ}$ patients. To date, vast numbers of clinical trials for

*Correspondence: Dr JW Young, Department of Psychiatry, University of California San Diego, 9500 Gilman Drive MC 0804, La Jolla, CA 92093-0804, USA, Tel: +0I 619543 3582, Fax: +01 6197359205 ,

E-mail: jaredyoung@ucsd.edu

${ }^{5}$ These authors contributed equally to this work.

${ }^{6}$ Present address: School of Pharmacy, University of Auckland, Auckland, New Zealand

${ }^{7}$ Present address: Lier Pharmacy, De Lier, The Netherlands

Received 7 July 20 16; revised 25 October 20 I6; accepted 4 November 2016; accepted article preview online 5 December 2016 psychiatric patients have failed at the cost of time, effort, billions of dollars, and the hope of the patients being tested. Many of these trials were based on positive preclinical data that failed to demonstrate efficacy in human trials. Failed human trials are often attributed to the lack of consistency in quantifying the same neural processes across species and the use of 'fast and dirty' behavioral techniques that have littleto-no relevance to human testing (Sarter, 2004). To that end, the National Institutes of Mental Health (NIMH) formed the Cognitive Neuroscience Treatment Research to Improve Cognition in Schizophrenia (CNTRICS) to identify behavioral paradigms that could be tested across species with translational validity (Carter and Barch, 2007; Dudchenko et al, 2013; Gilmour et al, 2013; Lustig et al, 2013) and promote the use of biomarkers of neural systems engaged during performance of these cross-species translational paradigms (Barch et al, 2012; Carter and Barch, 2012; Luck et al, 2011). An important initial step in such national initiatives is to establish what, if any, cognitive deficits and 
their associated biomarkers of neural system measures are apparent in putative cross-species translational paradigms.

The five-choice continuous performance test (5C-CPT), originally developed for testing in mice (Young et al, 2009), was highlighted by the CNTRICS initiative as a promising cross-species paradigm for assessing the control of attention (Lustig et al, 2013). Consistent with other human CPTs, the $5 \mathrm{C}-\mathrm{CPT}$ presents and requires responses to targets and inhibition of responses to non-target trials. Importantly, with more target than non-target trials, the 5C-CPT measures the control of attention, because it theoretically requires subjects to inhibit a prepotent response (Ford et al, 2004; Lustig et al, 2013), although to our knowledge, this has not yet been empirically demonstrated.

In terms of evidence for cross-species translational validity, we have observed the following in rodents and in humans: (a) $36 \mathrm{~h}$ sleep deprivation-induced deficits (van Enkhuizen et al, 2014); (b) amphetamine-induced improvement (MacQueen et al, in preparation); (c) parietal requirement for performance from human functional magnetic resonance imaging (fMRI) and rodent lesion studies (McKenna et al, 2013b); and (d) vigilance decrement observations across time (Young et al, 2013a; Young et al, 2009). Hence, consistent with a signal detection task, manipulation-induced changes in performance can be consistent across species (Bushnell et al, 2003). Importantly, the human 5C-CPT is also clinically sensitive, as patients with SZ exhibit deficient performance (Young et al, 2013a), consistent with other CPTs (Cornblatt and Keilp, 1994; Nuechterlein, 1991). Characterization of neural processing measures underlying normal and impaired behavioral performance of SZ patients in this cross-species task could therefore accelerate the development of procognitive therapeutics that target attentional systems, as drug effects observed on this task in animals may be more likely to span the translational bridge to human trials. fMRI studies can be conducted in the 5C-CPT (McKenna et al, 2013b), but such studies would prove difficult to conduct in rodents. In contrast, electroencephalographic (EEG) studies are possible in both humans (Bickel et al, 2012; Kleinlogel et al, 2007) and rodents (Brigman et al, 2013; Nagy et al, 2015), although knowledge of EEG response dynamics in patients with $\mathrm{SZ}$ is required before adaptation for rodent models (Featherstone et al, 2015; Gandal et al, 2010).

Although numerous studies have examined biomarker/ attentional performance in impaired neuropsychiatric patients via the examination of event-related potential (ERP) responses to infrequent target oddball stimuli (eg, $\mathrm{N}_{200}$ or $\mathrm{N}_{2}$ and $P_{300}$ or $P_{3}$ (Turetsky et al, 2015)), such oddball tasks are rarely, if ever, used in clinical neuropsychological assessments of patient populations. Conversely, computerized CPTs are frequently used in clinical evaluations, but relatively few studies have assessed the neural substrates of cognitive control using translatable CPTs, given the relative dearth of available tasks with cross-species validity. Previous studies found decreased ERP amplitudes in patients with SZ and their first-degree relatives, in particular late amplitudes of posterior parietal regions $\left(P_{3}\right)$, and augmented early amplitudes over frontal regions (anterior $N_{100}$ or $N_{1}$ ) (Sponheim et al, 2006), with reduced $P_{3}$ amplitudes in patients with SZ (Clementz et al, 2008; Knott et al, 1999) and in children at risk (Friedman et al, 1986). Interestingly, when $P_{3}$ amplitudes over midline scalp sensors were examined in response to target and non-target trials, the lack of amplitude differences by stimulus type in patients correctly classified all patients and controls (Knott et al, 1999). Although target and non-target ERP scalp topographies are fairly consistent across studies, testing modality (auditory $v s$ visual) can alter ERP amplitude and latency differences relevant to the interpretations of sensory and attentional functioning seen in SZ (Tekok-Kilic et al, 2001; Morales-Muñoz et al, 2016). Source localization analyses of high-density EEG recordings during CPTs (Doehnert et al, 2010) may help resolve contradictory findings in earlier studies. This study aimed to characterize and determine the electrophysiological correlates of 5C-CPT performance in patients with $\mathrm{SZ}$ and healthy controls (HCs). We hypothesized that patients with SZ would exhibit the following: (1) impaired 5C-CPT performance; (2) altered difference wave ERP amplitudes (between target and non-target trials); and (3) altered source localizations relative to $\mathrm{HC}$ participants.

\section{MATERIALS AND METHODS}

\section{Participants}

SZ outpatients $(n=25)$, and gender and age-matched HCs $(n=26)$ participated in the current study (Table 1$)$. Written informed consent was obtained in accordance with University of California San Diego institutional review boardapproved procedures. Participants were assessed diagnostically using the Structured Clinical Interview for Diagnostic and Statistical Manual of Mental Disorders, Fourth Edition (First et al, 1995), the Scale for the Assessment of Negative

Table I Demographic Means and SD by Group for Age, Education Level, and Smoking Status (Smoker vs Non-Smoker) of Healthy Comparison Subjects $(\mathrm{HC})$ and Patients with SZ

\begin{tabular}{|c|c|c|c|}
\hline \multirow[t]{2}{*}{ Demographics } & \multicolumn{2}{|c|}{ Group means ( \pm SD, min-max) } & \multirow[t]{2}{*}{ p-va } \\
\hline & HC $(n=26)$ & $S Z(n=25)$ & \\
\hline Mean age (years) & $37.8( \pm|0.9,2|-55)$ & $41.4( \pm \mid 1.7,23-60)$ & NS \\
\hline Education & $14.6( \pm 2.2, \mid 1-19)$ & $13.4( \pm 2.3,7-18)$ & NS \\
\hline Sex (\% male) & $62 \%$ & $80 \%$ & NS \\
\hline Smoking & $31 \%$ & $36 \%$ & NS \\
\hline Right handedness & $92 \%$ & $80 \%$ & NS \\
\hline Age of onset (years) & & $21( \pm 6.4,10-40)$ & \\
\hline Illness duration (years) & & $22( \pm 10.5, \mid-37)$ & \\
\hline SAPS total score & & $6.5( \pm 4.0,0-13)$ & \\
\hline SANS total score & & $16.7( \pm 4.4,6-24)$ & \\
\hline \multirow[t]{5}{*}{ GAF } & & $42.2( \pm 6.9,36-73)$ & \\
\hline & $\begin{array}{c}\text { Patients receiving } \\
\text { medication type, no. }\end{array}$ & & \\
\hline & Typical (exclusively) & 5 & \\
\hline & Atypical (exclusively) & 18 & \\
\hline & Typical+atypical & 3 & \\
\hline
\end{tabular}

Abbreviations: HC, healthy control; NS, not significant; SANS, Scale for the Assessment of Negative Symptoms; SAPS, Scale for the Assessment of Positive Symptoms; SZ, schizophrenia.

No significant differences for any demographics were observed. 
a

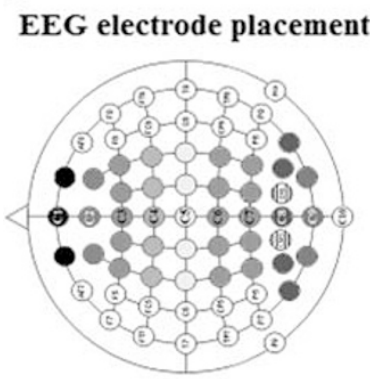

b

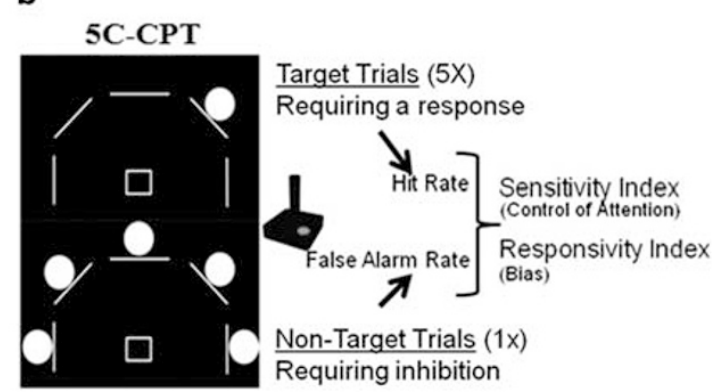

Figure I Task schematics. Schematic of centroid mapping indicated by number, calculated according to weighted averages of neighboring channels as indicated by color (a). Five-choice continuous performance task (5C-CPT) schematic (b), where target trials are presented by a single circle (top), requiring a response from the joystick in that direction (up and left in the example provided), whereas non-target trials are presented by five circles (below), requiring the inhibition of responding. Target trials contribute to the hit rate (HR) measurement, whereas non-target trials contribute to the false alarm rate (FAR) measurement (response inhibition). These measures are combined to produce the primary outcome measure for control of attention, the SI, whereas responsivity index measures bias of responding.

Symptoms (SANS; Andreasen, 1983), the Scale for the Assessment of Positive Symptoms (Andreasen, 1984), and the modified Global Assessment of Functioning Scale (Hall, 1995). SZ patients did not have an Axis I diagnosis other than SZ and HCs did not have any Axis I diagnosis. Exclusion criteria included drug abuse or dependence within the last 2 months, neurologic insult such as significant head trauma and/or loss of consciousness.

\section{Task: 5C-CPT}

Consistent with previous reports (Young et al, 2013a), the participants were given a brief practice on the task (see Supplementary Information). They were instructed to move the joystick in the direction a circle (target stimuli) appears, but inhibit from responding if five circles appeared simultaneously (non-target stimuli; Figure 1b). After a joystick response, the line under the selected stimulus flashed to indicate which target was selected, otherwise no other feedback was provided irrespective of accuracy. Stimuli were presented for $100 \mathrm{~ms}$ in a random order to reduce temporal predictability (Cope et al, 2016), with a $1 \mathrm{~s}$ response window available and a variable inter-trial intervals $(0.5,1$, or $1.5 \mathrm{~s})$. All participants understood the task and correctly performed the practice block before initiating the session. The full task consisted of 648 trials, 540 target stimuli, and 108 non-target stimuli, presented pseudorandomly so that no more than 3 presentations of a specific stimulus appeared consecutively.

Responses were recorded and include hits (correctly responding to a target stimulus) and misses (omissions, not responding to a target stimulus), incorrects (responding to locations other than the target), as well as false alarms (FAs; responding to non-target stimuli) and correct rejections (withholding from responding to non-target stimuli). Composite metrics of task performance were used in the analysis of performance, including hit rate (HR), and FA rate (FAR) as indicated in our previous work (Young et al, 2013a). The sensitivity and responsivity indices (measures of vigilance and bias respectively) were also calculated using signal detection theory (Green and Swets, 1966; McNicol, 1972), the former measures appropriate responding (Frey and Colliver, 1973) and the latter provides a measure of the 'tendency to respond' (bias).

\section{Electrophysiological Recording and Data Processing}

Continuous electrophysiological (EEG) data were recorded using a BioSemi Active Two system. During data acquisition the electrode offsets were kept below $25 \mathrm{mV}$ and all channels were referenced to the system's internal loop (CMS/DRL electrodes). Data were recorded in DC mode from 64 scalp leads, four electrooculogram leads recorded at the superior and inferior orbit of the left eye and outer canthi of each eye, one nose, and two mastoid electrodes for offline rereferencing. All data were collected using a $1048 \mathrm{~Hz}$ sampling rate using a first-order anti-aliasing filter and all preprocessing occurred offline using Brain Vision Analyzer 2.0 (Brain Products $\mathrm{GmbH}$ ). Bad channels were interpolated using a spherical spline interpolation and re-referenced to the average reference. Data were digitally band pass filtered between 1 and $70 \mathrm{~Hz}$ ( $24 \mathrm{db} / \mathrm{oct})$ using a Butterworth zero phase-shift filter with $48 \mathrm{db}$ /octave rolloff and eye movement artifacts were corrected using our established procedures. Epochs were generated from -100 to $700 \mathrm{~ms}$ post stimulus onset for correct trials. Only correct trials were used for ERP analysis due to the low number of task related errors. Epochs with additional EEG artifacts (adjacent sample amplitudes and/or max voltage changes exceeding $\pm 70 \mu \mathrm{V} / \mathrm{ms}$ ) were rejected and all remaining epochs were baseline corrected from -100 to $0 \mathrm{~ms}$. Separate ERP waveforms were generated for target and non-target trials.

Difference waves for each subject were generated by subtracting the target wave from the non-target wave. As in previous CPT ERP studies, centroids were calculated using the mean amplitude of channels centered on a midline channel and extending bi-laterally forming a strip of electrodes (eg, Centroid $3=$ Mean of $F_{z}, F_{1}, F_{2}, F_{3}, F_{4}$; Fallgatter et al, 2002 and Figure 1a). Based on this methodology, no-go anteriorization (NGA) values were generated and compared. Hence in addition, a priori ERP analyses focused on results from centroid peaks (Knott et al, 1999). Group-level grand average waveforms indicated three distinct time windows during which subject-level ERP peaks (point with greatest absolute maxima within a time window) were selected for statistical analysis, thought to represent early sensory components ((100-150 ms; $P_{100}$ or $P_{1}$; Herrmann and Knight, 2001), a middle latency transitional wave corresponding to response selection (150-250 ms; $N_{200}$ or $N_{2}$ ), and later temporal 
Centroid ERP Difference Waves, Analysis Time Windows, and Mean Response Times per Group

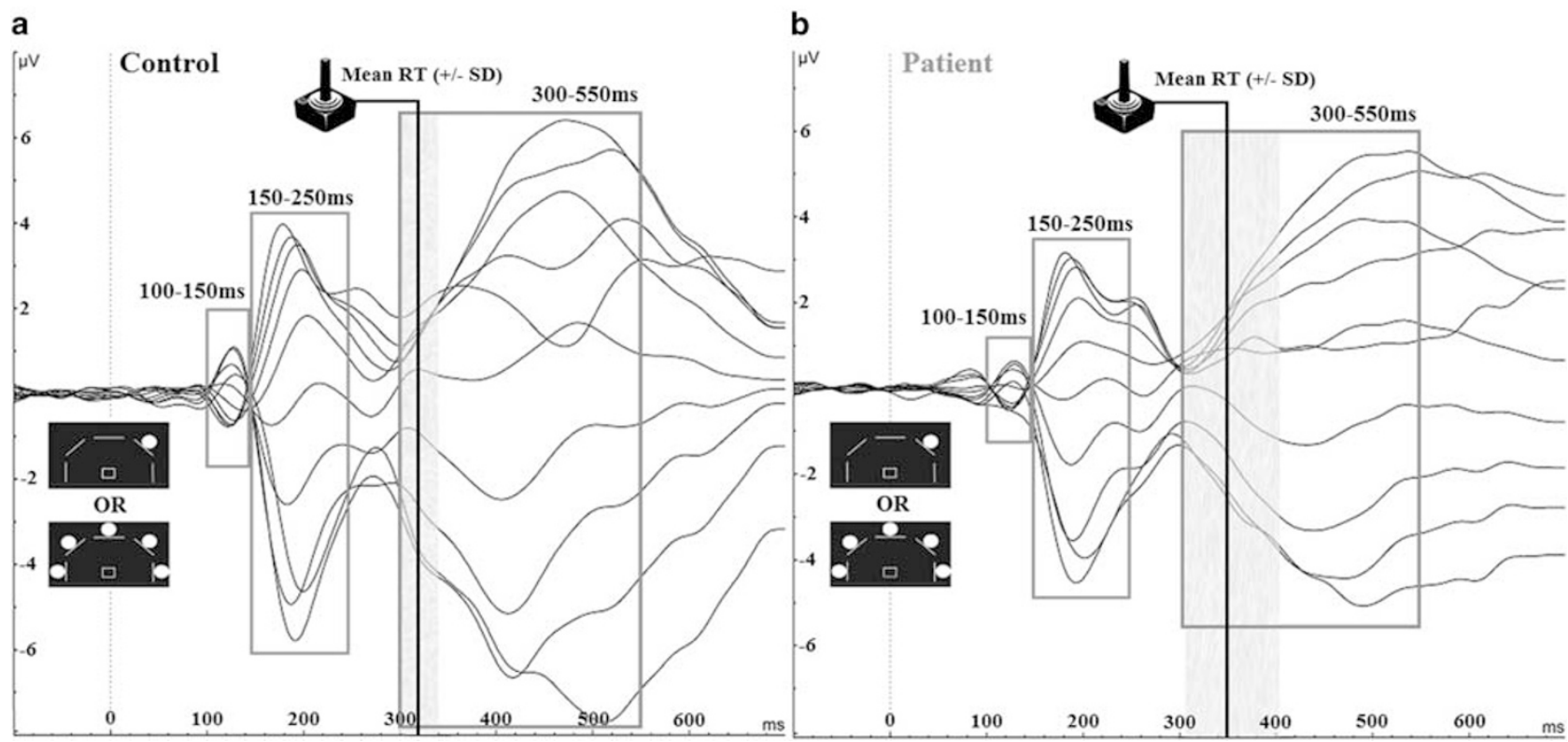

Figure 2 Electroencephalographic (EEG) butterfly plots. Butterfly plots of centroid difference waveforms and time windows for analysis (I00-I50, I50250, and 300-550 ms) for healthy comparison subjects (control; a) and schizophrenia patients (b). Vertical black lines indicate mean reaction time for each group with shaded areas indicating standard deviation.

window that corresponded to response action and visual feedback of choice (300-550 ms; $P_{300}$ or $P_{3}$; Figure 2).

\section{Source Analysis}

Source analysis of the ERP peaks was done in the Brainstorm environment using the Standardized low-resolution brain electromagnetic tomography algorithm (Pascual-Marqui, 2002; Tadel et al, 2011). A three-sphere head model was used and the source space was constrained to the cortex of a template brain with 3006 voxels (Berg and Scherg, 1994). The source image data was calculated at every time point for each subject. To test for group differences, the source image data for each subject was averaged across a $20 \mathrm{~ms}$ window centered over each of the three peaks for the group averaged ERP. Comparisons were done using clusterbased permutation statistics (1000 Monte Carlo simulations; (Maris and Oostenveld, 2007)). Separate source analyses were done for the target and non-target ERP waveforms. To compare with the centroid result, the same statistical comparisons were also done for the wider windows chosen for the ERPs.

\section{Statistical Analyses}

One-way multivariate analyses of variance (MANOVAs) and $\chi^{2}$-tests (where appropriate) were used to compare group differences in demographics, behavioral task performance, and ERP amplitudes across trial types, time windows, and centroids. Spearman's correlations were used to assess the relationships between behavioral performance metrics and ERP amplitudes, as well as symptom ratings within each group during each time window. Tukey post-hoc analyses of statistically significant or relevant main and interaction effects were performed where applicable, with Bonferroni corrections conducted for multiple comparisons. All data are reported as mean and SEM. The level of probability for statistical significance was set at 0.05 and for correlational analyses at 0.01 . All statistics were performed using SPSS (22.0, Chicago, USA).

\section{RESULTS}

\section{Demographics}

The demographics of SZ patients and healthy subjects are shown in Table 1. One-way ANOVAs demonstrated no significant between group differences in age, smoking status, level of education, or handedness (Fs $<1, N S$ ), and $\chi^{2}$ analysis indicated no significant between group gender distributions $(F(1)=2.09, p<0.13)$. These variables were not included in further analyses.

\section{C-CPT Behavioral Differences}

Patients with SZ exhibited significantly poorer overall task performance relative to HCs (sensitivity index (SI)). Patients also demonstrated significantly lower HRs, slower reaction times, and a higher FAR compared with HCs, but did not differ on response strategy (bias) as measured by responsivity index (Table 2 and Figure 3 insets).

\section{Task-Related ERPs}

Target trials. MANOVAs of centroid amplitude by group during target trials were completed for each time window. No significant differences for any centroid ERP amplitude between $\mathrm{HC}$ and SZ participants were observed during the early time window $(100-150 \mathrm{~ms} ; \quad F$ 's $<2.5, \quad p$ 's $>0.05$; Supplementary Figure 1). During the middle time window $(150-250 \mathrm{~ms})$, patients demonstrated significantly reduced 
Table 2 Between Group Comparisons of 5C-CPT Behavioral Performance of Healthy Comparison (HC) Subjects vs Patients with SZ

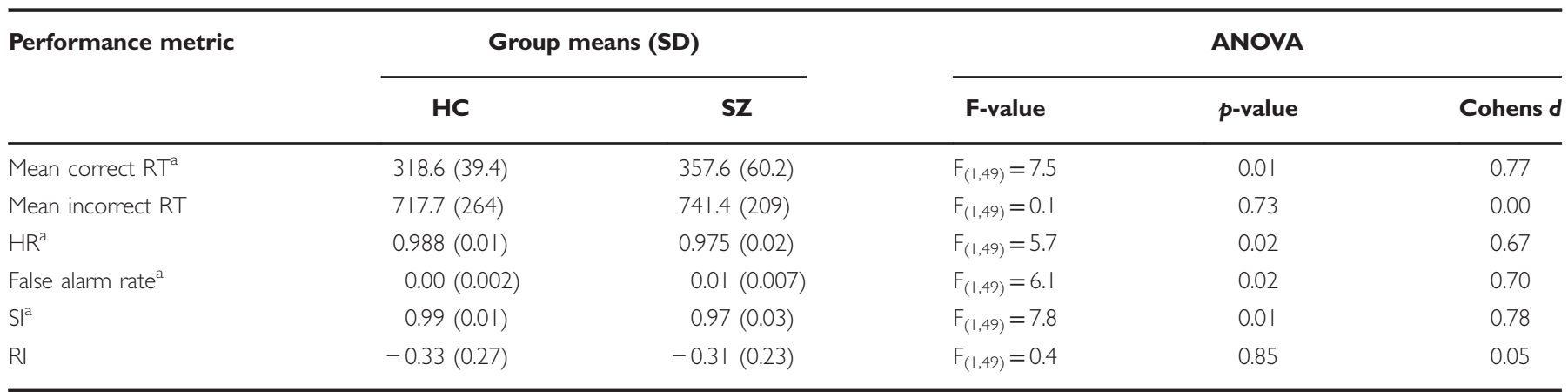

Abbreviations: 5C-CPT, five-choice continuous performance task; HC, healthy control; HR, hit rate; RI, responsivity index; SI, sensitivity index; SZ, schizophrenia. asignificant group differences.

Between Group ERP Waveforms and Task Performance for Target, Non-Target, and Difference Waves
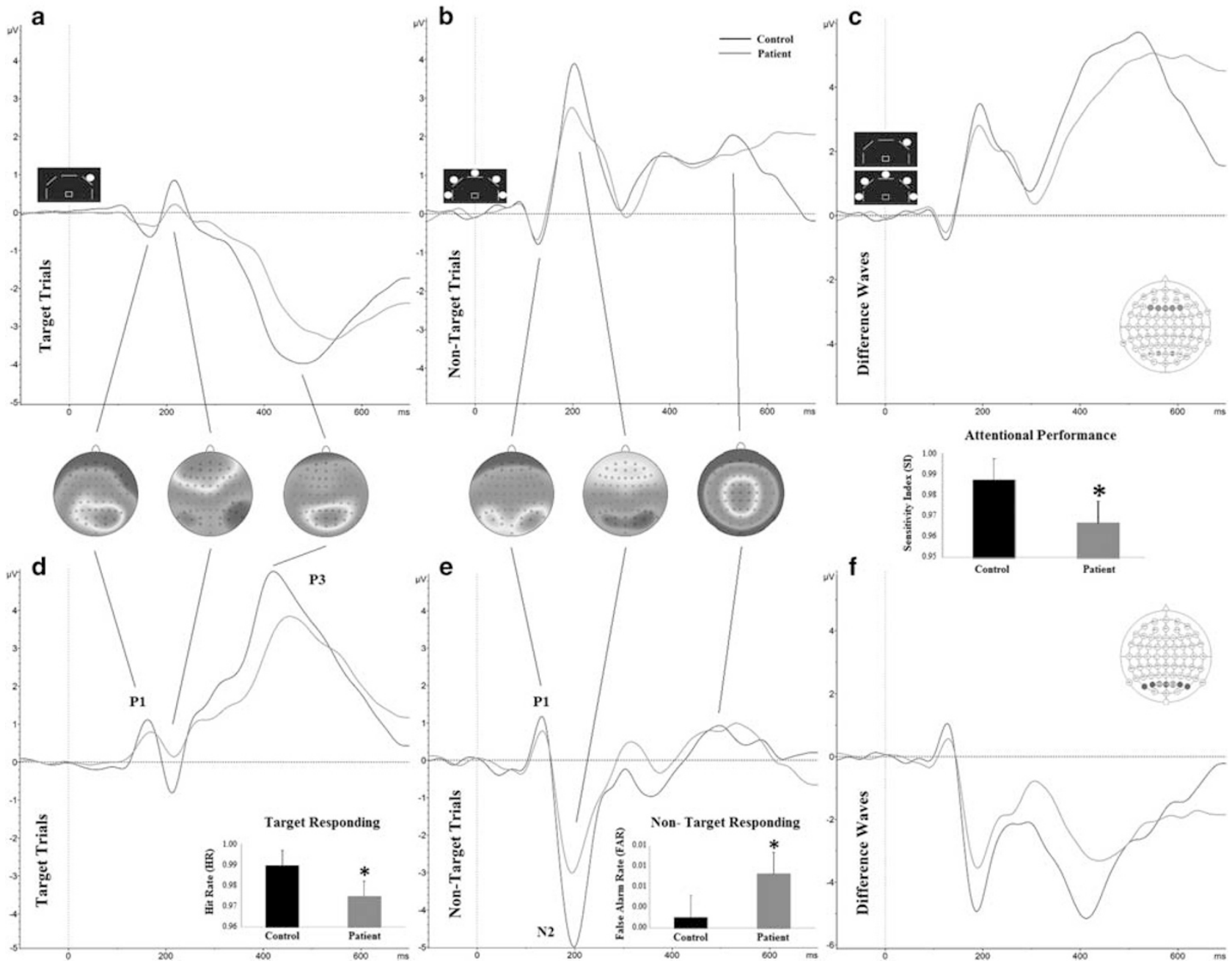

Figure 3 Event-related potential (ERP) and behavioral data. Between group ERP comparisons for target trials (a,d), non-target trials (b,e), and difference waves (c,f) measured at the frontal centroid 3 (top row) and the occipital parietal centroid 8 (bottom row) of healthy participants (control) and schizophrenia patients. The $P_{1}, N_{2}$, and $P_{3}$ ERPs are indicated. Inset topography plots show greatest scalp amplitude location for ERP peak. Inset bar graphs indicate between group behavioral performance within conditions, with schizophrenia patients exhibiting reduced target responding (hit rate (HR)), increased response disinhibition (false alarm rate), and impaired vigilance (SI). Behavioral data presented as mean+SEM, * $p<0.05$ compared with control. A full color version of this figure is available at the Neuropsychopharmacology journal online.

ERP amplitudes at centroids one $\left(\mathrm{F}_{(1,50)}=8.1, p<0.01\right)$, two $\left(\mathrm{F}_{(1,50)}=7.9, \quad p<0.01\right)$, three $\left(\mathrm{F}_{(1,50)}=6.5, \quad p<0.02\right)$, six $\left(\mathrm{F}_{(1,50)}=4.5, p<0.04\right)$, seven $\left(\mathrm{F}_{(1,50)}=6.4, p<0.02\right)$, eight $\left(\mathrm{F}_{(1,50)}=7.2, \quad p<0.01\right)$, and nine $\left(\mathrm{F}_{(1,50)}=5.6, \quad p<0.03\right.$;
Supplementary Figure 1). During the later time window (300-550 ms), SZ patients exhibited decreased ERP amplitude compared with controls at centroid eight $\left(\mathrm{F}_{(1,50)}=6.3\right.$, $p<0.02)$. 


\section{Centroid Difference Wave ERP Amplitude Comparisons}

a

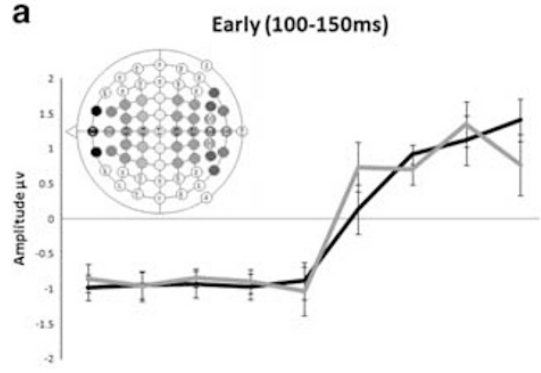

b Middle(150-250ms)

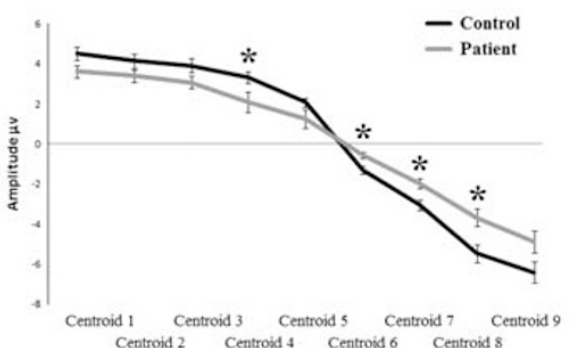

C

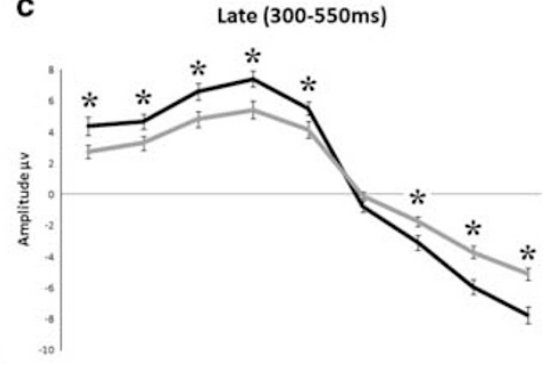

Figure 4 Centroid difference wave amplitudes. The between group centroid difference wave amplitudes and SEM for the early ( $P_{1}$ : I00-150 ms; a), mid ( $\mathrm{N}_{2}$ : 150-250 ms; b), and late ( $P_{3}:$ 300-550 ms; c), sensory time window post stimulus onset for healthy comparison subjects (control in black) and schizophrenia patients (in red). ${ }^{*} p<0.05$ compared with controls. A full color version of this figure is available at the Neuropsychopharmacology journal online.

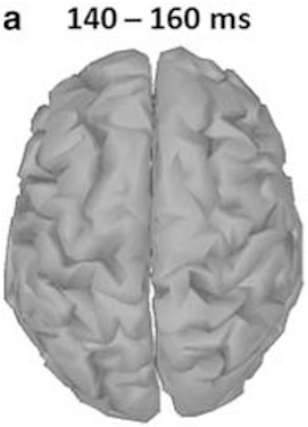

(N.A.)

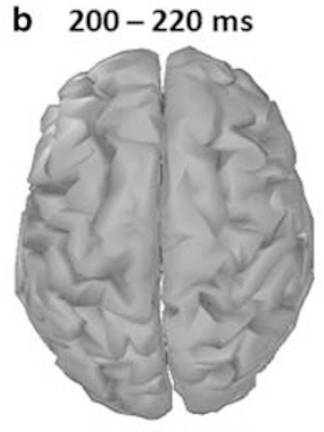

Left DLPFC
C $\quad 390-410 \mathrm{~ms}$

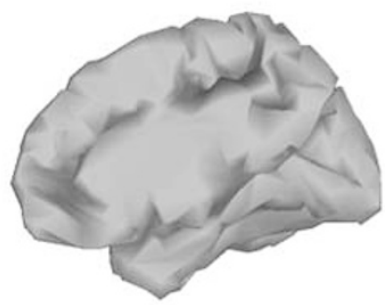

Right Posterior Cingulate

Figure 5 Electroencephalographic (EEG) source analysis for target trials. Cortical maps indicate sources for event-related potential (ERP) peak activation during each time window. No difference between schizophrenia patients and healthy comparison participants were observed early time window $\left(P_{1}\right.$; a). Source localization analyses of the middle time window $\left(\mathrm{N}_{2}\right)$ indicated significantly greater positive source activity (current source/efferent dipole), in the dorsolateral prefrontal cortex (DLPFC) for healthy participants compared to schizophrenia patients (b). Source localization analyses also indicated greater negative source activity (current sink/afferent dipole) in the right posterior cingulate cortex in the later time window $\left(P_{3}\right)$ for healthy participants compared with schizophrenia patients (c). Source analyses were computed for the $20 \mathrm{~ms}$ ( $10 \mathrm{~ms}$ pre and post) surrounding the group-level ERP peak amplitude for that time window. For both $\mathrm{N}_{2}$ and $P_{3}$ windows, healthy participants demonstrated greater source activity compared to schizophrenia patients, with source colors representing dipole direction of significant between group differences. A full color version of this figure is available at the Neuropsychopharmacology journal online.

Non-target trials. During the early time window (100$150 \mathrm{~ms})$, SZ patients demonstrated increased ERP amplitude only at centroid six $\left(\mathrm{F}_{(1,50)}=4.1, p<0.05\right.$; Supplementary Figure 2). During the middle time window (150-250 ms), SZ patients demonstrated significantly reduced ERP amplitudes at centroids one $\left(\mathrm{F}_{(1,50)}=4.9, p<0.04\right)$, two $\left(\mathrm{F}_{(1,50)}=4.2\right.$, $p<0.05)$, six $\left(\mathrm{F}_{(1,50)}=5.2, p<0.03\right)$, seven $\quad\left(\mathrm{F}_{(1,50)}=7.8\right.$, $p<0.01)$, eight $\left(\mathrm{F}_{(1,50)}=6.1, p<0.02\right)$, and nine $\left(\mathrm{F}_{(1,50)}=4.1\right.$, $p<0.05)$ with centroid three at trend level $\left(\mathrm{F}_{(1,50)}=4.8\right.$, $p<0.06$; Supplementary Figure 2). During the later time window (300-550 ms), patients demonstrated decreased ERP amplitude only at centroid nine $\left(\mathrm{F}_{(1,50)}=5.4, p<0.03\right)$.

Difference waves between target and non-target trials. MANOVAs of centroid difference wave amplitudes by group was completed for each time window. No significant between group differences in the earliest (100-150 ms) time window were seen $(\mathrm{F}$ 's $<2.5, p$ 's $>0.05$; Figure $4 \mathrm{a})$. During the middle (150-250 ms) time window however, SZ demonstrated significantly smaller difference wave amplitudes at centroids four $\left(\left(\mathrm{F}_{(1,50)}=4.5, p<0.04\right)\right.$, six $\left(\mathrm{F}_{(1,50)}=7.6, p<0.01\right)$, seven $\left(\mathrm{F}_{(1,50)}=9.1, p<0.01\right)$, and eight $\left(\mathrm{F}_{(1,50)}=8.1, p<0.01\right)$;
Figure $4 \mathrm{~b})$. During the later (300-550 ms) time window, SZ patients demonstrated significantly smaller difference wave amplitudes at centroids one $\left(\mathrm{F}_{(1,50)}=5.2, p<0.03\right)$, two $\left(\mathrm{F}_{(1,50)}=4.2, p<0.05\right)$, three $\left(\mathrm{F}_{(1,50)}=5.4, p<0.03\right)$, four $\left(\mathrm{F}_{(1,50)}=7.1, p<0.01\right)$, five $\left(\mathrm{F}_{(1,50)}=4.1, p<0.05\right)$, seven $\left(\mathrm{F}_{(1,50)}=5.2, p<0.03\right)$, eight $\left(\mathrm{F}_{(1,50)}=13.5, p<0.00\right)$, and nine $\left(\mathrm{F}_{(1,50)}=15.6, p<0.00\right)$; Figure $\left.4 \mathrm{c}\right)$. No NGA differences were observed between groups (see Supplementary Information and Supplementary Figure 1).

Correlating ERP analyses with behavioral measurements in the later response action time window (300$550 \mathrm{~ms}$ ). Spearman's correlational analyses were performed to assess the relationships between behavioral performance indices and difference wave ERP amplitudes within the later time window. As difference waves were calculated based on target $v s$ non-target ERPs and SI reflected the behavioral differences between target and non-target performance (HRFAR), we predicted correlations between these measures. Consistent with our prediction, SI correlated with ERPs at centroid eight $(\rho=-0.489, p<0.0001)$. When analyzed by group, HC displayed a significant correlation between SI and 
amplitudes only at centroid eight $(\rho=-0.523, p<0.008)$, with SZ patients not exhibiting significant correlations.

\section{Source Level Results}

With the $20 \mathrm{~ms}$ average centered over each peak, source data comparison between SZ patients and HC revealed brain regions of significant differences for the second $\left(N_{2}\right)$ and third $\left(P_{3}\right)$ peaks of the target condition (cluster corrected $p<0.05)$. Significant differences were found in the left dorsolateral prefrontal cortex (DLPFC) for the second peak and in the right posterior cingulate cortex for the third peak (Figure 5). The results indicate that the source current density is more positive in HC than SZ patients for the second peak, but less in HC than SZ patients for the third peak. The scout time traces in the localized regions indicate that in both cases, patients had a decrease in signal amplitude. When averaged across the same time windows as in the centroid analysis, the source level statistics demonstrated similar patterns of change without correction (Supplementary Figures 2 and 3).

\section{Exploratory Correlational Analyses}

Although these results indicate a biomarker for attentional functioning in SZ, prior research suggests the medial-frontal $P_{3}$ may be particularly sensitive to negative symptomotology (Kawasaki et al, 2007). Spearman's correlational analyses were performed to assess the relationships between negative symptoms (as measured by the SANS) and centroid $P_{3}$ difference-wave amplitudes across the scalp. The Bonferroni correction was used to correct for multiple comparisons, resulting in an $\alpha$ of 0.005 . Significant negative relationships were found between $P_{3}$ amplitude and SANS total score at fronto-central centroids three $(r=-0.628, p<0.001)$ and four $(r=-0.742, p<0.000)$ with similar relationships seen at parieto-occipital centroids eight and nine ( $r$ 's $>0.456$, ps $<0.02$ ). However, the parieto-occipital relationships failed to survive correction (Supplementary Figure 4).

\section{DISCUSSION}

This study replicates and extends our previous behavioral characterization of 5C-CPT performance deficits in SZ patients (Young et al, 2013a). Using ERPs, the present study demonstrated that SZ patients exhibit robust information processing abnormalities even on trials with correct behavioral performance. These abnormalities were localized to regions known to be involved in attentional network, ie, the DLPFC and posterior cingulate cortex (Squire et al, 2013). Moreover, these ERP abnormalities were significantly correlated with behavioral performance metrics-stronger in $\mathrm{HC}$ than SZ participants-suggesting that impaired stimulus-related information processing may still significantly contribute to subsequent attentional performance deficits.

Patients with SZ exhibited poorer 5C-CPT behavioral performance than $\mathrm{HC}$ subjects, driven in part by reduced responses to target stimuli, reproducing earlier reports (Young et al, 2013a). In addition, significantly higher nontarget responses (indicating response disinhibition of SZ patients) were also observed $v s$ a nonsignificant increase previously (Young et al, 2013a), possibly as a result of a larger sample size in this study. Hence, consistent with other CPTs (Cornblatt and Keilp, 1994; Cornblatt and Malhotra, 2001; Nuechterlein, 1991), SZ patients exhibited consistently significantly deficient responses in the 5C-CPT irrespective of trial type, not driven simply by an altered response strategy (bias). These behavioral differences are significant with medium to large effect sizes (Table 2), despite a high standard of performance of participants in this task. In addition, the clinical significance of such differences have yet to be determined using standard metrics. In terms of $5 \mathrm{C}$ CPT performance level however, the observed level is not at ceiling given that we have observed significant improvements in human task performance based on motivation, amphetamine, or modafinil (MacQueen et al, in preparation; Cope et $\mathrm{al}$, in preparation; Bismark et al, in preparation). In order to provide varying levels of performance however, a 5C-CPT variant is under development using two levels of trial-type difficulty. This adapted 5C-CPT will also determine whether neural responses reported here will vary based upon difficulty level.

Importantly, the present study identified neural measures of deficient performance of SZ patients. Thus, the $5 \mathrm{C}-\mathrm{CPT}$ is a compelling probe for quantifying prepotent responses, because of evidence of a higher frontal non-target $P_{3}$ compared with target $P_{3}$ in $\mathrm{HC}$ subjects (Figure 3; Ford et al, 2004; Pfefferbaum et al, 1985), indicative of assessing the control of attention (Luck et al, 2012; Lustig et al, 2013), as opposed to conflict monitoring (Gonzalez-Rosa et al, 2013). This stronger non-target frontal ERP response is also consistent with our fMRI observations of strong frontal activation during non-target trials (McKenna et al, 2013a). Larger amplitude target $P_{3}$ s compared with non-target $P_{3} \mathrm{~s}$ were observed in occipital/parietal regions in subjects performing the 5C-CPT. Importantly, SZ patients exhibited weaker neurophysiological responses compared to HCs irrespective of trial type, particularly in mid and late $P_{3}$ time windows, supporting the behavioral data, indicating inattentive/response disinhibition of patients. This effect has been observed previously and suggests that SZ patients did not develop a prepotent response as described (Chun et al, 2013; Ford et al, 2004). This pattern of deficits was not however seen in patients with bipolar disorder (Chun et al, 2013). In addition, frontal and occipital/parietal non-target $N_{2}$ waves were also observed, which were also weaker in SZ patients compared with healthy subjects. Interestingly, posterior $P_{1}$ (early time window $100-150 \mathrm{~ms}$ ) were observed in response to both target and non-target trials consistent with other CPTs (Sponheim et al, 2006). The $P_{1}$ was consistent between trial types and is linked to responses in the visual area. This ERP was the only one that that did not differ between patients with SZ and healthy comparison subjects, consistent with other observations (Sponheim et al, 2006), supporting that visual processing of the stimuli was unaffected by the one (target) $v s$ five (non-target) visual stimuli and was unaffected in patients. Hence, patients with SZ exhibited weaker ERPs at $N_{2}$ and $P_{3}$ peaks with stronger frontal non-target $v s$ target $P_{3}$ s observed, whereas the opposite was true for occipital/parietal $P_{3}$ s irrespective of disease state. Among patients, lower amplitude frontocentral ERPs were related to negative symptoms, with similar but nonsignificant relationships demonstrated at occipital/ 
parietal centroids. These findings are consistent with altered ERP networks in response to target $v s$ non-target stimuli (Wynn et al, 2015).

The primary biomarker of impaired performance of SZ patients appeared to be the deficient $N_{2}$ ERP, as reduced peaks were observed irrespective of target and non-target trials or in frontal or occipital/parietal regions. $P_{1}$ peaks occur in response to the visual stimuli presented (targets and non-targets), with $\mathrm{N}_{2}$ occurring after visual processing prior to choice selection $\left(P_{3} s\right)$. As ERPs were calculated in response to from correct responses, it is evident that $\mathrm{N}_{2}$ deficiencies in SZ patients occur as a result of altered information processing reflecting impaired response selections. In fact, the elevated non-target $v s$ target $\mathrm{N}_{2}$ amplitude may provide evidence of the cognitive control required for this task.

Reduced $\mathrm{N}_{2}$ amplitudes have been recorded in SZ patients performing attentional tasks from auditory (Salisbury et al, 1994; Umbricht et al, 2006) and visual (Alain et al, 1998; Bruder et al, 1998) stimuli. Importantly, first-episode SZ patients exhibit reduced $N_{2}$ amplitudes (Umbricht et al, 2006), which differ from generalized attentional deficits, as patients with posttraumatic stress disorder exhibit elevated $\mathrm{N}_{2}$ peaks (Galletly et al, 2008). As noted above, the temporal window of information processing reflected in the $\mathrm{N}_{2}$ during attentive processing coincides with middle latency ERPs evoked during passive auditory oddball paradigms reflecting automatic sensory discrimination (ie, mismatch negativity; Light et al, 2015). Source analysis of the $N_{2}$ suggest that the most significant deficit is localized to the left DLPFC, which has been shown to be a key brain region involved in attention and cognitive functioning, particularly in the attentional network (Squire et al, 2013). The neural circuitry of this key region has also been consistently shown to be abnormal in SZ patients during information processing (Barch et al, 2001). The present study thus further underscores the contribution of impairments in this transitional window from stimulus registration, which appears to be largely intact in SZ, but cascade forward to the cognitive $\left(N_{2}\right)$ and functional sequelae of the illness.

Source level differences were also observed for the $P_{3}$ of the target condition. SZ patients exhibited decreased activity (ie, decreased amplitude of negative peak), in the posterior cingulate cortex during target trials in the $P_{3}$ window. The posterior cingulate cortex has been linked to the modulation of attention and conscious awareness (Leech and Sharp, 2014). Given that this activation occurred around the time subjects reacted, this effect could include the feedback they were given in the task. For SZ patients, abnormal posterior cingulate cortical activity was linked both anatomically and functionally via studies showing decreased white matter integrity of the cingulate fasciculus and decreased metabolic activity in the cingulate gyrus (Haznedar et al, 2004; Kubicki et al, 2003). The decreased source level activity observed herein is both consistent with previous findings and supportive of further development of $\mathrm{N}_{2}$ differences during 5C-CPT as a translational assay for treatment development.

Smoking status can impact ERPs (Knott et al, 1999) and cognitive performance, exerting modest attentional improvements in patients but deleteriously affecting healthy subjects when large sample sizes $(>100)$ are examined (Hahn et al, 2012). Smoking status was controlled for in the current study with approximately $36 \%$ of SZ and $31 \%$ of HC participants were smokers. Smoking status did not interact with any performance measure or ERP responses in the present study and the $N_{2}$ and $P_{3}$ differences observed provide sufficient neural response parameters that were unaffected by smoking status. Other CPT EEG studies have been conducted, but the $5 \mathrm{C}-\mathrm{CPT}$ used in the present study offers several advantages to alternative CPTs such as the AX-CPT, CPT-IP, and DSCPT. First, the 5C-CPT does not include additional cognitive/perceptual demands on performance such as working memory, number matching, or challenging visual perception (Silverstein et al, 1998). In addition, the 5C-CPT uses internationally recognized shapes as opposed to culturespecific stimuli, hence, in addition to being a domain pure assessment of control of attention, the task can also conducted in rodents (Barnes et al, 2012a, b; Harms et al, 2012; Tomlinson et al, 2014; Turner et al, 2013; van Enkhuizen et al, 2014; Young et al, 2013a; Young et al, 2009; Young et al, 2013b; Young et al, 2011), enabling more relevant cross-species assessment (Young and Geyer, 2015). This translational utility further increases the value of the behavioral and neurophysiological biomarkers investigated during the current study.

These data support our premise that the 5C-CPT measures the control of attention (suppression of a prepotent response (Ford et al, 2004; Luck et al, 2012; Lustig et al, 2013). Importantly, difference wave amplitudes supported reduced ERP amplitudes at fronto-central and parietal centroids during $P_{3}$ and $N_{2}$ in SZ patients compared with HC subjects. Finally, correlations between centroid ERPs and primary $5 \mathrm{C}$ CPT performances was observed overall, but with stronger links seen to $\mathrm{HC}$ performance compared to SZ patients. Hence, the present study provides biomarkers of impaired control of attention of patients with SZ in the reversetranslated 5C-CPT.

In summary, we observed impaired 5C-CPT performance in patients with SZ compared with healthy subjects. These deficits could have arisen from reduced $N_{2}$ amplitudes irrespective of region or stimulus type, in SZ patients. Reduced target and non-target $P_{3}$ s were also observed in patients with SZ. These biomarkers of attentional deficits are more striking given that these ERPs were taken from only correct trials. Hence, even when responding correctly, patients with SZ do not exhibit as strong a neural response to trials compared to healthy subjects. The temporal precision of EEG and the comparable $P_{1}$ ERPs of patients and controls support the premise that the downstream neural processing of stimuli is weaker in patients with SZ compared to healthy subjects. Despite group differences already observed, future clinical studies would benefit from identifying commonalities of genes or environment. Preclinical studies could then use that information to create a more complete model of deficits in control of attention (Keeler and Robbins, 2011; Lustig et al, 2013; Young and Geyer, 2015; Young et al, 2012). With the capability of conducting EEG behavioral studies in mice (Brigman et al, 2013; Featherstone et al, 2015; Ji et al, 2013), genetic, environmental, paradigmatic, and EEG biomarker models (Luck et al, 2012) could be combined to enhance targeted therapeutic development (Markou et al, 2009). 


\section{FUNDING AND DISCLOSURE}

In the past 3 years Dr Young and Light's work has been funded by NIDA and NIMH, as well as the US Veteran's Administration VISN 22 Mental Illness, Research, Education, and Clinical Center. Dr Young has also received funding from Cerca Insights and Lundbeck, and has received consulting compensation for Amgen and honoraria from Arena Pharmaceuticals and Sunovian. Dr Light has received consulting from Forum, Astellas, and Boehringer-Ingelheim. Drs Bismark, McIlwain, Mr Grootendorst and Sun, and Ms Zhang report no extra funding sources. This work was supported by NIH grants R01-MH104344, UH2-MH109168, the Sidney R. Baer Jr. Foundation, Brain and Behavioral Research Foundation, and the Clinical Translational Research Institute (CTRI).

\section{REFERENCES}

Alain C, Hargrave R, Woods DL (1998). Processing of auditory stimuli during visual attention in patients with schizophrenia. Biol Psychiatry 44: 1151-1159.

Andreasen NC (1983). Scale for the Assessment of Negative Symptoms (SANS). University of Iowa: Iowa City.

Andreasen NC (1984). Scale for the Assessment of Positive Symptoms (SAPS). University of Iowa: Iowa City.

Barch DM, Carter CS, Braver TS, Sabb FW, MacDonald A, Noll DC et al (2001). Selective deficits in prefrontal cortex function in medication-naive patients with schizophrenia. Arch Gen Psychiatry 58: 280-288.

Barch DM, Moore H, Nee DE, Manoach DS, Luck SJ (2012). CNTRICS imaging biomarkers selection: Working memory. Schizophr Bull 38: 43-52.

Barnes SA, Young JW, Neill JC (2012a). D(1) receptor activation improves vigilance in rats as measured by the 5-choice continuous performance test. Psychopharmacology (Berl) 220: 129-141.

Barnes SA, Young JW, Neill JC (2012b). Rats tested after a washout period from sub-chronic PCP administration exhibited impaired performance in the 5-Choice Continuous Performance Test (5CCPT) when the attentional load was increased. Neuropharmacology 62: 1432-1441.

Berg P, Scherg M (1994). A fast method for forward computation of multiple-shell spherical head models. Electroencephalogr Clin Neurophysiol 90: 58-64.

Bickel S, Dias EC, Epstein ML, Javitt DC (2012). Expectancy-related modulations of neural oscillations in continuous performance tasks. Neuroimage 62: 1867-1876.

Bismark AW, Serences J, Young JW (in prepration) Psychometrics of the human $5 \mathrm{C}-\mathrm{CPT}$.

Brigman JL, Daut RA, Wright T, Gunduz-Cinar O, Graybeal C, Davis MI et al (2013). GluN2B in corticostriatal circuits governs choice learning and choice shifting. Nat Neurosci 16: 1101-1110.

Bruder G, Kayser J, Tenke C, Rabinowicz E, Friedman M, Amador X et al (1998). The time course of visuospatial processing deficits in schizophrenia: an event-related brain potential study. J Abnorm Psychol 107: 399-411.

Bushnell PJ, Benignus VA, Case MW (2003). Signal detection behavior in humans and rats: a comparison with matched tasks. Behav Processes 64: 121-129.

Carter CS, Barch DM (2007). Cognitive neuroscience-based approaches to measuring and improving treatment effects on cognition in schizophrenia: the CNTRICS initiative. Schizophr Bull 33: 1131-1137.

Carter CS, Barch DM (2012). Imaging biomarkers for treatment development for impaired cognition: report of the sixth
CNTRICS meeting: Biomarkers recommended for further development. Schizophr Bull 38: 26-33.

Chun J, Karam ZN, Marzinzik F, Kamali M, O'Donnell L, Tso IF et al (2013). Can P300 distinguish among schizophrenia, schizoaffective and bipolar I disorders? An ERP study of response inhibition. Schizophr Res 151: 175-184.

Clementz BA, Wang J, Keil A (2008). Normal electrocortical facilitation but abnormal target identification during visual sustained attention in schizophrenia. J Neurosci 28: 13411-13418.

Cope ZA, Halberstadt AL, van Enkhuizen J, Flynn AD, Breier M, Swerdlow NR et al (2016). Premature responses in the five-choice serial reaction time task reflect rodents' temporal strategies: evidence form the no-light and pharmacological challenges. Psychopharmacology 233: 13-25.

Cornblatt BA, Keilp JG (1994). Impaired attention, genetics, and the pathophysiology of schizophrenia. Schizophr Bull 20: 31-46.

Cornblatt BA, Malhotra AK (2001). Impaired attention as an endophenotype for molecular genetic studies of schizophrenia. Am J Med Genet 105: 11-15.

Doehnert M, Brandeis D, Imhof K, Drechsler R, Steinhausen HC (2010). Mapping attention-deficit/hyperactivity disorder from childhood to adolescence-no neurophysiologic evidence for a developmental lag of attention but some for inhibition. Biol Psychiatry 67: 608-616.

Dudchenko PA, Talpos J, Young J, Baxter MG (2013). Animal models of working memory: a review of tasks that might be used in screening drug treatments for the memory impairments found in schizophrenia. Neurosci Biobehav Rev 37: 2111-2124.

Fallgatter AJ, Aranda DR, Bartsch AJ, Herrmann MJ (2002). Longterm reliability of electrophysiologic response control parameters. J Clin Neurophysiol 19: 61-66.

Featherstone RE, McMullen MF, Ward KR, Bang J, Xiao J, Siegel SJ (2015). EEG biomarkers of target engagement, therapeutic effect, and disease process. Ann N Y Acad Sci 1344: 12-26.

First M, Spitzer R, Gibbon M, Williams J (1995). Structured Clinical Interview for DSMIV Axis 1. Disorders - Patient Edition. New York State Psychiatric Institute: New York.

Ford JM, Gray M, Whitfield SL, Turken AU, Glover G, Faustman WO et al (2004). Acquiring and inhibiting prepotent responses in schizophrenia: event-related brain potentials and functional magnetic resonance imaging. Arch Gen Psychiatry 61: 119-129.

Frey PW, Colliver JA (1973). Sensitivity and responsibility measures for discrimination learning. Learn Motivation 4: 327-342.

Friedman D, Cornblatt B, Vaughan H Jr., Erlenmeyer-Kimling L (1986). Event-related potentials in children at risk for schizophrenia during two versions of the continuous performance test. Psychiatry Res 18: 161-177.

Galletly CA, McFarlane AC, Clark R (2008). Differentiating cortical patterns of cognitive dysfunction in schizophrenia and posttraumatic stress disorder. Psychiatry Res 159: 196-206.

Gandal MJ, Edgar JC, Ehrlichman RS, Mehta M, Roberts TP, Siegel SJ (2010). Validating gamma oscillations and delayed auditory responses as translational biomarkers of autism. Biol Psychiatry 68: 1100-1106.

Gilmour G, Arguello A, Bari A, Brown VJ, Carter C, Floresco SB et al (2013). Measuring the construct of executive control in schizophrenia: defining and validating translational animal paradigms for discovery research. Neurosci Biobehav Rev 37: 2125-2140.

Gonzalez-Rosa JJ, Inuggi A, Blasi V, Cursi M, Annovazzi P, Comi G et al (2013). Response competition and response inhibition during different choice-discrimination tasks: evidence from ERP measured inside MRI scanner. Int J Psychophysiol 89: 37-47.

Green DM, Swets JA (1966). Signal Detection Theory and Psychophysics. Wiley \& Sons: New York. 
Green MF (1996). What are the functional consequences of neurocognitive deficits in schizophrenia? Am J Psychiatry 153: 321-330.

Green MF, Nuechterlein KH, Kern RS, Baade LE, Fenton WS, Gold JM et al (2008). Functional co-primary measures for clinical trials in schizophrenia: results from the MATRICS Psychometric and Standardization Study. Am J Psychiatry 165: 221-228.

Hahn C, Hahn E, Dettling M, Gunturkun O, Ta TM, Neuhaus AH (2012). Effects of smoking history on selective attention in schizophrenia. Neuropharmacology 62: 1897-1902.

Hall RC (1995). Global assessment of funcitoning: a modified scale. Pscyhosomatics 36: 267-275.

Harms LR, Turner KM, Eyles DW, Young JW, McGrath JJ, Burne TH (2012). Attentional processing in C57BL/6J mice exposed to developmental vitamin D deficiency. PLoS One 7: e35896.

Haznedar MM, Buchsbaum MS, Hazlett EA, Shihabuddin L, New A, Siever LJ (2004). Cingulate gyrus volume and metabolism in the schizophrenia spectrum. Schizophr Res 71: 249-262.

Herrmann CS, Knight RT (2001). Mechanisms of human attention: event-related potentials and oscillations. Neurosci Biobehav Rev 25: 465-476.

Ji B, Wang X, Pinto-Duarte A, Kim M, Caldwell S, Young JW et al (2013). Prolonged ketamine effects in hypomorphic mice: mimicking phenotypes of schizophrenia. PLoS ONE 8: e66327.

Kawasaki Y, Sumiyoshi T, Higuchi Y, Ito T, Takeuchi M, Kurachi M (2007). Voxel-based analysis of P300 electrophysiological topography associated with positive and negative symptoms of schizophrenia. Schizophrenia Res 94: 164-171.

Keefe RS, Bilder RM, Davis SM, Harvey PD, Palmer BW, Gold JM et al (2007). Neurocognitive effects of antipsychotic medications in patients with chronic schizophrenia in the CATIE Trial. Arch Gen Psychiatry 64: 633-647.

Keeler JF, Robbins TW (2011). Translating cognition from animals to humans. Biochem Pharmacol 81: 1356-1366.

Kleinlogel H, Strik W, Begre S (2007). Increased NoGoanteriorisation in first-episode schizophrenia patients during continuous performance test. Clin Neurophysiol 118: 2683-2691.

Knott V, Mahoney C, Labelle A, Ripley C, Cavazzoni P, Jones B (1999). Event-related potentials in schizophrenic patients during a degraded stimulus version of the visual continuous performance task. Schizophr Res 35: 263-278.

Kubicki M, Westin CF, Nestor PG, Wible CG, Frumin M, Maier SE et al (2003). Cingulate fasciculus integrity disruption in schizophrenia: a magnetic resonance diffusion tensor imaging study. Biol Psychiatry 54: 1171-1180.

Leech R, Sharp DJ (2014). The role of the posterior cingulate cortex in cognition and disease. Brain 137(Pt 1): 12-32.

Light GA, Swerdlow NR, Thomas ML, Calkins ME, Green MF, Greenwood TA et al (2015). Validation of mismatch negativity and $\mathrm{P3a}$ for use in multi-site studies of schizophrenia: characterization of demographic, clinical, cognitive, and functional correlates in COGS-2. Schizophr Res 163: 63-72.

Luck SJ, Ford JM, Sarter M, Lustig C (2012). CNTRICS final biomarker selection: control of attention. Schizophr Bull 38: 53-61.

Lustig C, Kozak R, Sarter M, Young JW, Robbins TW (2013). CNTRICS final animal model task selection: control of attention. Neurosci Biobehav Rev 37: 2099-2110.

Maris E, Oostenveld R (2007). Nonparametric statistical testing of EEG- and MEG-data. J Neurosci Methods 164: 177-190.

Markou A, Chiamulera C, Geyer MA, Tricklebank M, Steckler T (2009). Removing obstacles in neuroscience drug discovery: the future path for animal models. Neuropsychopharmacology 34: 74-89.

McKenna B, Young JW, Dawes SE, Asgaard GL, Eyler LT (2013a). Bridging the bench to bedside gap: validation of a reversetranslated rodent continuous performance test using functional magnetic resonance imaging. Psychiatry Res Neuroimaging 212: $183-191$ in press.
McKenna BS, Young JW, Dawes SE, Asgaard GL, Eyler LT (2013b). Bridging the bench to bedside gap: validation of a reversetranslated rodent continuous performance test using functional magnetic resonance imaging. Psychiatry Res 212: 183-191.

McNicol D (1972). A Primer of Signal Detection Theory. George Allen \& Unwin: London.

Morales-Muñoz I, Jurado-Barba R, Fernández-Guinea S, Rodríguez-Jiménez R, Jiménez-Arriero MÁ, Criado JR et al (2016). Sensory gating deficits in first-episode psychosis: evidence from neurophysiology, psychophysiology, and neuropsychology. J Nervous Mental Dis 204: 877-884.

Nagy LR, Featherstone RE, Hahn CG, Siegel SJ (2015). Delayed emergence of behavioral and electrophysiological effects following juvenile ketamine exposure in mice. Transl Psychiatry 5: e635.

Nuechterlein KH (1991). Vigilance in schizoprhenia and related disorders. In: Steinhauer SR, Gruzelier JH, Zubin J (eds). Neuropsychology, Psychophysiology, and Information Processing. Hanbook of Schizophrenia. Elsevier Science: New York, Vol 5, pp 397-433.

Parks JJ, Radke AQ, Tandon R (2008). Impact of the CATIE findings on state mental health policy. Psychiatr Serv 59: 534-536.

Pascual-Marqui RD (2002). Standardized low-resolution brain electromagnetic tomography (sLORETA): technical details. Methods Find Exp Clin Pharmacol 24(Suppl D): 5-12.

Pfefferbaum A, Ford JM, Weller BJ, Kopell BS (1985). ERPs to response production and inhibition. Electroencephalogr Clin Neurophysiol 60: 423-434.

Salisbury DF, O'Donnell BF, McCarley RW, Shenton ME, Benavage A (1994). The N2 event-related potential reflects attention deficit in schizophrenia. Biol Psychol 39: 1-13.

Sarter M (2004). Animal cognition: defining the issues. Neurosci Biobehav Rev 28: 645-650.

Silverstein SM, Light G, Palumbo DR (1998). The sustained attention test: a measure of attentional disturbance. Computers Hum Behav 14: 463.

Sponheim SR, McGuire KA, Stanwyck JJ (2006). Neural anomalies during sustained attention in first-degree biological relatives of schizophrenia patients. Biol Psychiatry 60: 242-252.

Squire RF, Noudoost B, Schafer RJ, Moore T (2013). Prefrontal contributions to visual selective attention. Annu Rev Neurosci 36: 451-466.

Tadel F, Baillet S, Mosher JC, Pantazis D, Leahy RM (2011). Brainstorm: a user-friendly application for MEG/EEG analysis. Comput Intell Neurosci 2011: 879716.

Tekok-Kilic A, Shucard JL, Shucard DW (2001). Stimulus modality and Go/NoGo effects on P3 during parallel visual and auditory continuous performance tasks. Psychophysiology 38: 578-589.

Tomlinson A, Grayson B, Marsh S, Harte MK, Barnes SA, Marshall KM et al (2014). Pay attention to impulsivity: modelling low attentive and high impulsive subtypes of adult ADHD in the 5 -choice continuous performance task $(5 \mathrm{C}-\mathrm{CPT})$ in female rats. Eur Neuropsychopharmacol 24: 1371-1380.

Turetsky BI, Dress EM, Braff DL, Calkins ME, Green MF, Greenwood TA et al (2015). The utility of P300 as a schizophrenia endophenotype and predictive biomarker: clinical and sociodemographic modulators in COGS-2. Schizophr Res 163: 53-62.

Turner KM, Young JW, McGrath JJ, Eyles DW, Burne TH (2013). Cognitive performance and response inhibition in developmentally vitamin D (DVD)-deficient rats. Behav Brain Res 242: 47-53.

Umbricht DS, Bates JA, Lieberman JA, Kane JM, Javitt DC (2006). Electrophysiological indices of automatic and controlled auditory information processing in first-episode, recent-onset and chronic schizophrenia. Biol Psychiatry 59: 762-772.

van Enkhuizen J, Acheson D, Risbrough V, Drummond S, Geyer MA, Young JW (2014). Sleep deprivation impairs performance in the 
5-choice continuous performance test: similarities between humans and mice. Behav Brain Res 261: 40-48.

Wynn JK, Jimenez AM, Roach BJ, Korb A, Lee J, Horan WP et al (2015). Impaired target detection in schizophrenia and the ventral attentional network: Findings from a joint event-related potentialfunctional MRI analysis. Neuroimage Clin 9: 95-102.

Young JW, Geyer MA (2015). Developing treatments for cognitive deficits in schizophrenia: the challenge of translation. J Psychopharmacol 29: 178-196.

Young JW, Geyer MA, Rissling AJ, Sharp RF, Eyler LT, Asgaard GL et al (2013a). Reverse translation of the rodent 5C-CPT reveals that the impaired attention of people with schizophrenia is similar to scopolamine-induced deficits in mice. Transl Psychiatry 3: e324.
Young JW, Light GA, Marston HM, Sharp R, Geyer MA (2009). The 5-choice continuous performance test: evidence for a translational test of vigilance for mice. PLoS ONE 4: e4227.

Young JW, Meves JM, Geyer MA (2013b). Nicotinic agonistinduced improvement of vigilance in mice in the 5-choice continuous performance test. Behav Brain Res 240: 119-133.

Young JW, Powell SB, Geyer MA (2012). Mouse pharmacological models of cognitive disruption relevant to schizophrenia. Neuropharmacology 62: 1381-1390.

Young JW, Powell SB, Scott CN, Zhou X, Geyer MA (2011). The effect of reduced dopamine D4 receptor expression in the 5-choice continuous performance task: Separating response inhibition from premature responding. Behav Brain Res 222: 183-192.

Supplementary Information accompanies the paper on the Neuropsychopharmacology website (http://www.nature.com/npp) 